\title{
Ruang Norm-2 dan Ruang Hasil Kali Dalam-2
}

\author{
J.Manuhutu, Y.A.Lesnussa, H. Batkunde \\ JurusanMatematika Fakultas MIPA UniversitasPattimura \\ Jln. Ir. M. Putuhena, Kampus Unpatti, Poka-Ambon \\ e-mail: johnmanuhutu@yahoo.com,yopi_a_lesnussa@yahoo.com,
}

\begin{abstract}
ABSTRAK
Konsep ruang norm- $n(n \geq 2)$ pertama kali diperkenalkan oleh Gähler, sementara ruang hasil kali dalam-2 dikembangkan oleh Misiak. Tulisan ini akan membahas mengenai ruang norm-2 dan ruang hasil kali dalam2 secara umum. Selain itu, makalah ini juga akan meninjau lebih jauh tentang intepretasi geometri dari ruang norm-2 juga hubungan antara ruang norm-2 dan ruang hasil kali dalam-2.
\end{abstract}

Kata Kunci:Ruang norm-2, Ruang Hasil Kali Dalam-2.

\section{ABSTRACT}

The concept of $n$-normed spaces $(n \geq 2)$ was originally introduced by Gähler, while the 2-inner product spaces developed by Misiak. This paper will discuss about 2-normed spaces and 2-inner product spaces generally. In addition, this paper will review interpretation of the geometry of space norm-2 further and also the relationship between 2-normed spaces and 2-inner product spaces.

Keywords: 2-normed spaces, 2-inner product spaces

\section{Pendahuluan}

Konsep ruang norm pertama kali dikemukakan oleh S. Banach, H. Hahn dan N. Weiner pada tahun 1922, yang lebih lanjut dikembangkan oleh S. Banach pada tahun 1932. Ruang norm adalah ruang-ruang yang dibangun dari ruang vektor dengan diDefinisikan norm didalamnya. Ruang norm-2 (juga perumuman ruang norm- $n$ untuk $(n \geq 2)$ ), pertama kali dikemukakan oleh S. Gähler pada tahun 1960-an. Setelah itu Misiak mengembangkan ruang hasil kali dalam- $n$ (untukn $\geq 2$ ) pada tahun 1989. (lihat Gahler [3,4] dan Misiak [8]).

Ruang norm adalah suatu ruang vektor yang dilengkapi oleh suatu norm yang didefinisikan di dalamnya (Kreyszig, [2]). Jika norm suatu vector dapat diinterpretasikan sebagai panjang dari suatu vektor, maka norm-2 dapat diinterpretasikan sebagai luasan jajar genjang yang direntang oleh dua vektor.

Konsep ruang norm-n merupakan perumuman dari ruang norm-2. Sedangkan norm- $n$ dapat diintepretasikansebagai volume pararel epipedium yang direntang oleh $n$ buah vektor (Batkunde [1] dan Gunawan [5,6]). Sama halnya dengan ruang norm, banyak aspek yang dapat dikaji pada ruang norm-2, yang akan ditinjau pada bagian selanjutnya.

\section{Metode Penelitian}

Penelitian ini disusun dengan menggunakan metode studi pustaka yang diperoleh dari buku, jurnal maupun sumber online, pendalaman literatur serta wawasan dasar yang dimiliki penulis. 


\section{Hasil Dan Pembahasan}

Perhatikan bahwa jika $(X,\langle.,\rangle$.$) merupakan suatu ruang hasil kali dalam, maka ketaksamaan$ Cauchy-Schwarz di $X$ dapat dinyatakan sebagai:

$$
\left|\begin{array}{ll}
\langle x, x\rangle & \langle x, y\rangle \\
\langle y, x\rangle & \langle y, y\rangle
\end{array}\right| \geq 0 \quad ; \forall x, y \in X
$$

Perhatikan juga bahwa, jika terdapat dua vektor $x, y \in X$, maka

$$
A(x, y)=\left|\begin{array}{ll}
\langle x, x\rangle & \langle x, y\rangle \\
\langle y, x\rangle & \langle y, y\rangle
\end{array}\right|^{\frac{1}{2}}
$$

Menyatakan luas jajaran genjang yang direntang oleh dua vektor $x$ dan $y$, akan ditunjukkan kemudian bahwa $A(x, y)$ merupakan suatu norm-2.

Berikut penjabaran lebih lanjut tentang ruang norm-2 dan ruang hasil kali dalam-2.

\subsection{Ruang Norm-2 dan Ruang Hasil Kali Dalam-2}

Definisi 1.

Misal $X$ dengan $\operatorname{dim}(X) \geq 2$. Pemetaan $\|.,\|:. X \times X \rightarrow \mathbb{R}$ yang memenuhi sifat:

i. $\|x, y\| \geq 0 ; \forall x, y \in X$

$\|x, y\|=0$ jika dan hanya jika $x, y$ bergantung linier.

ii. $\|x, y\|=\|y, x\| ; \forall x, y \in X$

iii. $\|\alpha x, y\|=|\alpha|\|x, y\|, \forall x, y \in X$

iv. $\|x+y, z\| \leq\|x+z\|+\|y+z\| ; \forall x, y, z \in X$.

Merupakan norm-2 pada $X$, dan pasangan $(X,\|.,\|$.$) disebut ruang norm-2.$

Contoh 2.

Perhatikan bahwa $\left(\mathbb{R}^{2},\langle\cdot\rangle,\right)$ merupakan ruang hasil kali dengan hasil kali dalamnya didefinisikan oleh:

dengan norm-2 didefinisikan oleh

$$
\langle x, y\rangle=x_{1} y_{1}+x_{2} y_{2}, \quad \forall x, y \in \mathbb{R}^{2}
$$

$$
\|x, y\|_{s}=\left|\begin{array}{ll}
\langle x, x\rangle & \langle x, y\rangle \\
\langle y, x\rangle & \langle y, y\rangle
\end{array}\right|^{\frac{1}{2}}
$$

Dapat diperiksa bahwa $\left(\mathbb{R}^{2},\|\cdot, \cdot\|\right)$ merupakan suatu ruang norm-2.

Contoh 3.

$\mathbb{R}^{d}$ dengan norm-2 yang didefiniskan oleh

Merupakan suatu ruang norm-2.

$$
\|x, y\|_{s}=\left(\frac{1}{2} \sum_{i=1}^{d} \sum_{j=1}^{d}\left|\begin{array}{ll}
x_{i} & x_{j} \\
y_{i} & y_{j}
\end{array}\right|^{2}\right)^{\frac{1}{2}}
$$


Selanjutnya jika adaluasan yang dibentuk oleh dua vector $x$ dan $y$, ternyata luasan yang dibentuk oleh vektor $x, y$ akan sama dengan luasan yang dibentuk oleh vektor $(x+\alpha y)$ dan $y$ yang dinyatakan dalam proposisi berikut.

Proposisi 4.

$\operatorname{Diberikan}(X,\|\cdot, \cdot\|)$ adalah suatu ruang norm-2, maka $\forall x, y \in X$ berlaku:

Bukti:

$$
\|x+\alpha y, y\|=\|x, y\| \quad, \alpha \in \mathbb{R}
$$

Perhatikan bahwa untuk $x, y \in X$, maka berlaku

Sebaliknya:

$$
\|x+\alpha y, y\| \leq\|x, y\|+|\alpha|\|y, y\|
$$

$$
\leq\|x, y\|
$$

$$
\leq\|x+\alpha y, y\|
$$

$$
\begin{gathered}
\|x, y\|=\|x+\alpha y-\alpha y, y\| \\
\leq\|x+\alpha y, y\|+|\alpha|\|y, y\|
\end{gathered}
$$

Dari (1) dan (2), diperoleh,

$\|x+\alpha y, y\|=\|x, y\| \quad, \forall x, y \in X, \alpha \in \mathbb{R}$.

\section{Definisi5.}

Misalkan $X$ ruang vektor. Maka pemetaan $\langle\because \cdot \mid \cdot\rangle: X \times X \times X \rightarrow \mathbb{R}$, yang bersifat:

i. $\langle x, x \mid z\rangle \geq 0 \quad, \forall x, z \in X$.

$\langle x, x \mid z\rangle=0$ jika dan hanya jika $x$ dan $z$ bergantung linier.

ii. $\langle x, x \mid z\rangle=\langle z, z \mid x\rangle, \forall x, z \in X$.

iii. $\langle x, y \mid z\rangle=\langle y, x \mid z\rangle, \forall x, y, z \in X$.

iv. $\langle\alpha x, y \mid z\rangle=\alpha\langle x, y \mid z\rangle, \forall x, y, z \in X, \alpha \in \mathbb{R}$.

v. $\left\langle x+x^{\prime}, y \mid z\right\rangle=\langle x, y \mid z\rangle+\left\langle x^{\prime}, y \mid z\right\rangle \quad ; \forall x, x^{\prime}, y, z \in X$.

Merupakan hasil kali dalam-2 pada $X$, dan pasangan $(X,\langle\because \cdot \mid \cdot\rangle)$ merupakan ruang hasil kali dalam-2.

Contoh 6.

Perhatikan bahwa $\left(\mathbb{R}^{\mathrm{n}},\langle\because \cdot \mid \cdot\rangle\right)$ dengan $n \in \mathbb{N}$, merupakan ruang hasil kali dalam-2 dengan hasil kali dalam-2 yang didefinisikan sebagai :

$$
\langle x, y \mid z\rangle=\left|\begin{array}{ll}
\langle x, y\rangle & \langle x, z\rangle \\
\langle z, y\rangle & \langle z, z\rangle
\end{array}\right| ; x, y, z \in \mathbb{R}^{\mathrm{n}}
$$

Teorema 7.

$\operatorname{Misalkan}(X,\langle\because \mid \cdot\rangle)$ adalah ruang hasil kali dalam-2, maka $\forall x, y, z \in X$ berlaku Ketaksamaan Cauchy-Schwarz, yakni:

Bukti:

$$
|\langle x, y \mid z\rangle| \leq\langle x, x \mid z\rangle^{\frac{1}{2}}\langle y, y \mid z\rangle^{\frac{1}{2}}
$$

Perhatikan bahwa untuk $x, y, z \in X$ dan $\forall \alpha \in \mathbb{R}$,

Atau dapat ditulis

$$
\langle\alpha x+y, \alpha x+y \mid z\rangle \geq 0,
$$

$$
\langle\alpha x, \alpha x \mid z\rangle+\langle y, \alpha x \mid z\rangle+\langle\alpha x, y \mid z\rangle+\langle y, y \mid z\rangle \geq 0,
$$


menghasilkan

$$
\alpha^{2}\langle x, x \mid z\rangle+2 \alpha\langle x, y \mid z\rangle+\langle y, y \mid z\rangle \geq 0
$$

Persamaan di atas merupakan suatu persamaan kuadrat yang tak negatif, sehingga menghasilkan

atau

$$
4\langle x, y \mid z\rangle^{2}-4\langle x, x \mid z\rangle\langle y, y \mid z\rangle \leq 0,
$$

dengan kata lain,

$$
\langle x, y \mid z\rangle^{2} \leq\langle x, x \mid z\rangle\langle y, y \mid z\rangle,
$$

$$
|\langle x, y \mid z\rangle| \leq\langle x, x \mid z\rangle^{\frac{1}{2}}\langle y, y \mid z\rangle^{\frac{1}{2}}
$$

\subsection{Hubungan Ruang Norm-2 dan Ruang Hasil Kali Dalam-2.}

Teorema 8.

Misal $(X,\langle\because \mid \cdot\rangle)$ ruang hasil kali dalam-2, maka

$$
\|x, y\|=\langle x, x \mid y\rangle^{\frac{1}{2}}
$$

Merupakan norm-2 pada $X$ (dapat diperiksa oleh pembaca).

Lebih lanjut dalam ruang hasil kali dalam-2, dengan norm-2 yang diinduksi oleh hasil kali dalam-2, berlaku hukum jajar genjang, yaitu:

Teorema 9.

Bukti:

$$
\|x+y, z\|^{2}+\|x-y, z\|^{2}=2\left(\|x, z\|^{2}+\|y, z\|^{2}\right) ; \forall x, y, z \in X
$$

Untuk $x, y, z \in X$ maka dapat dituliskan,

Atau dengan kata lain

$$
\|x+y, z\|^{2}+\|x-y, z\|^{2}=\langle x+y, x+y \mid z\rangle+\langle x-y, x-y \mid z\rangle
$$

$$
\|x+y, z\|^{2}+\|x-y, z\|^{2}=(\langle x, x \mid z\rangle+\langle y, x \mid z\rangle)+(\langle x, x \mid z\rangle-\langle y, x \mid z\rangle)-(\langle x, y \mid z\rangle-\langle y, y \mid z\rangle),
$$

Yang menghasilkan

atau

$$
\|x+y, z\|^{2}+\|x-y, z\|^{2}=2\langle x, x \mid z\rangle+2\langle y, y \mid z\rangle,
$$

$$
\|x+y, z\|^{2}+\|x-y, z\|^{2}=2\left(\|x, z\|^{2}+\|y, z\|^{2}\right) .
$$

Sebagai akibatnya, dalam ruang hasil kali dalam-2, dengan norm-2 yang diinduksi oleh hasil kali dalam-2, berlaku kesamaan polarisasi, yaitu:

Akibat10.

Sama dengan hukum jajar genjang. $\forall x, y, z \in X$ dalam ruang hasil kali dalam-2 juga berlaku kesamaan polarisasi, yaitu:

Bukti:

$$
\langle x, y \mid z\rangle=\frac{1}{4}\left(\|x+y, z\|^{2}-\|x-y, z\|^{2}\right)
$$

Untuk $x, y, z \in X$ maka dapat dituliskan,

$$
\|x+y, z\|^{2}-\|x-y, z\|^{2}=(\langle x+y, x+y \mid z\rangle-\langle x-y, x-y \mid z\rangle)
$$

atau

$$
\|x+y, z\|^{2}-\|x-y, z\|^{2}=(\langle x, x \mid z\rangle+2\langle x, y \mid z\rangle+\langle y, y \mid z\rangle)-(\langle x, x \mid z\rangle-2\langle x, y \mid z\rangle+\langle y, y \mid z\rangle),
$$

Dan menghasilkan

dengan kata lain

$$
\|x+y, z\|^{2}-\|x-y, z\|^{2}=4\langle x, y \mid z\rangle
$$

$$
\langle x, y \mid z\rangle=\frac{1}{4}\left(\|x+y, z\|^{2}-\|x-y, z\|^{2}\right) .
$$


Selanjutnya akan ditinjau ketaksamaan yang setara dengan Ketaksamaan Cauchy-Schwarz (teorema 7) pada kasus standar.

Teorema 11.

Ketaksamaan Cauchy-Schwarz pada kasus standar di ruang hasil kali dalam-2 setara dengan:

Bukti:

$$
\left|\begin{array}{lll}
\langle x, x\rangle & \langle x, y\rangle & \langle x, z\rangle \\
\langle y, x\rangle & \langle y, y\rangle & \langle y, z\rangle \\
\langle z, x\rangle & \langle z, y\rangle & \langle z, z\rangle
\end{array}\right| \geq 0 ; \forall x, y, z \in \mathbb{R}^{n} ; n \in \mathbb{N}
$$

Perhatikan bahwa; $\forall x, y, z \in \mathbb{R}^{n} ; n \in \mathbb{N}$ berlaku:

dimana

$$
\left|\begin{array}{ccc}
\langle x, x\rangle & \langle x, y\rangle & \langle x, z\rangle \\
\langle y, x\rangle & \langle y, y\rangle & \langle y, z\rangle \\
\langle z, x\rangle & \langle z, y\rangle & \langle z, z\rangle
\end{array}\right|=\left|\begin{array}{ccc}
\left\langle x^{\prime}, x\right\rangle & \left\langle x^{\prime}, y\right\rangle & \left\langle x^{\prime}, z\right\rangle \\
\left\langle y^{\prime}, x\right\rangle & \left\langle y^{\prime}, y\right\rangle & \left\langle y^{\prime}, z\right\rangle \\
\langle z, x\rangle & \langle z, y\rangle & \langle z, z\rangle
\end{array}\right|
$$

dapat dijabarkan menjadi

$$
\left|\begin{array}{ccc}
\left\langle x^{\prime}, x\right\rangle & \left\langle x^{\prime}, y\right\rangle & \left\langle x^{\prime}, z\right\rangle \\
\left\langle y^{\prime}, x\right\rangle & \left\langle y^{\prime}, y\right\rangle & \left\langle y^{\prime}, z\right\rangle \\
\langle z, x\rangle & \langle z, y\rangle & \langle z, z\rangle
\end{array}\right| \geq 0
$$

$$
\left\langle x^{\prime}, x^{\prime}\right\rangle\left|\begin{array}{cc}
\left\langle y^{\prime}, y^{\prime}\right\rangle & \left\langle y^{\prime}, z\right\rangle \\
\left\langle z, y^{\prime}\right\rangle & \langle z, z\rangle
\end{array}\right|-\left\langle x^{\prime}, y^{\prime}\right\rangle\left|\begin{array}{cc}
\left\langle y^{\prime}, x^{\prime}\right\rangle & \left\langle y^{\prime}, z\right\rangle \\
\left\langle z, x^{\prime}\right\rangle & \langle z, z\rangle
\end{array}\right|+\left\langle x^{\prime}, z\right\rangle\left|\begin{array}{cc}
\left\langle y^{\prime}, x^{\prime}\right\rangle & \left\langle y^{\prime}, y^{\prime}\right\rangle \\
\left\langle z, x^{\prime}\right\rangle & \left\langle z, y^{\prime}\right\rangle
\end{array}\right| \geq 0,
$$

atau

dengan kata lain

$$
\left\langle x^{\prime}, x^{\prime}\right\rangle\left|\begin{array}{cc}
\left\langle y^{\prime}, y^{\prime}\right\rangle & 0 \\
0 & \langle z, z\rangle
\end{array}\right|-\left\langle x^{\prime}, y^{\prime}\right\rangle\left|\begin{array}{cc}
\left\langle y^{\prime}, x^{\prime}\right\rangle & 0 \\
0 & \langle z, z\rangle
\end{array}\right| \geq 0,
$$

$$
\left\langle x^{\prime}, y^{\prime}\right\rangle\left\langle y^{\prime}, x^{\prime}\right\rangle\langle z, z\rangle \leq\left\langle x^{\prime}, x^{\prime}\right\rangle\left\langle y^{\prime}, y^{\prime}\right\rangle\langle z, z\rangle,
$$

yang dapat dituliskan sebagai

atau

$$
\left\langle x^{\prime}, y^{\prime}\right\rangle^{2} \leq\left\langle x^{\prime}, x^{\prime}\right\rangle\left\langle y^{\prime}, y^{\prime}\right\rangle
$$

sehingga dapat dituliskan menjadi

$$
\left\langle x^{\prime}, y^{\prime}\right\rangle \leq\left\langle x^{\prime}, x^{\prime}\right\rangle^{\frac{1}{2}}\left\langle y^{\prime}, y^{\prime}\right\rangle^{\frac{1}{2}},
$$

$$
\frac{|\langle x, y \mid z\rangle|}{\|x, z\|\|y, z\|} \leq 1 \Leftrightarrow|\langle x, y \mid z\rangle| \leq\|x, z\|\|y, z\|,
$$

dengan demikian menghasilkan

$$
|\langle x, y \mid z\rangle| \leq\langle x, x \mid z\rangle^{\frac{1}{2}}\langle y, y \mid z\rangle^{\frac{1}{2}} .
$$

\subsection{Interpretasi Geometri}

Pada ruang norm Euclid, hasil kali dalam (Euclidian inner product) antara dua vektor $u$ dan $v$ dinotasikan dengan $u \cdot v$, dalam hubungannya dengan norm data dituliskan sebagai:

$$
u \cdot v=\|u\|\|v\| \cos \theta
$$

dengan $\|u\|$ adalah panjang vektor $\mathrm{u},\|v\|$ adalah panjang vektor $\mathrm{v}$ dan $\theta$ adalah besar sudut antara dua vektor tersebut. 
Jika $u \neq 0$ dan $v \neq 0$ maka berdasarkan definisi diatas diperoleh:

sehingga dapat disimpulkan bahwa pada ruang norm-2

$$
\cos \theta=\frac{u \cdot v}{\|u\|\|v\|}
$$

$$
\cos \theta=\frac{\langle x, y \mid z\rangle}{\|x, z\|\|y, z\|}
$$

dengan $\theta$ menyatakan sudut yang dibentuk oleh bidang yang direntang oleh $x$ dan $z$ serta bidang yang di rentang oleh $y$ dan $z$ dan $\langle x, y \mid z\rangle$ menyatakan hasil kali dalamnya.

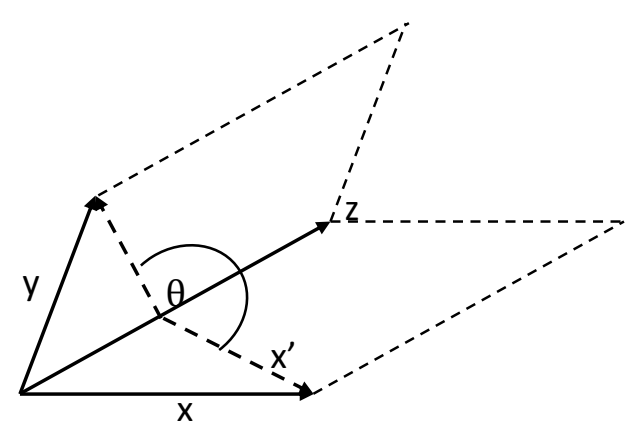

Gambar 1. Sudut $\theta$ yang dibentuk oleh bidang yang direntang oleh $x$ dan $z$ serta bidang yang di rentang oleh $y$ dan $z$

Pada kasus standar, perhatikan bahwa untuk $\mathrm{x}^{\prime} \perp \mathrm{z}$ dan $\mathrm{y}^{\prime} \perp \mathrm{z}\left(\mathrm{x}^{\prime}\right.$ merupakan proyeksi vektor $\mathrm{x}^{\prime}$ pada $\mathrm{z}$ dan y'merupakan proyeksi vektor y pada z) berlaku:

$\langle x, y \mid z\rangle=\left|\begin{array}{cc}\langle x, y\rangle & \langle x, z\rangle \\ \langle z, y\rangle & \langle z, z\rangle\end{array}\right|=\left|\begin{array}{cc}\left\langle x^{\prime}, y^{\prime}\right\rangle & \left\langle x^{\prime}, z\right\rangle \\ \left\langle z, y^{\prime}\right\rangle & \langle z, z\rangle\end{array}\right|=\left\langle x^{\prime}, y^{\prime}\right\rangle\|z\|^{2}$,

dan

$$
\|\mathrm{x}, \mathrm{z}\|=\left|\begin{array}{cc}
\langle\mathrm{x}, \mathrm{x}\rangle & \langle\mathrm{x}, \mathrm{z}\rangle \\
\langle\mathrm{z}, \mathrm{x}\rangle & \langle\mathrm{z}, \mathrm{z}\rangle
\end{array}\right|^{\frac{1}{2}}=\left|\begin{array}{cc}
\left\langle\mathrm{x}^{\prime}, \mathrm{x}^{\prime}\right\rangle & \left\langle\mathrm{x}^{\prime}, \mathrm{z}\right\rangle \\
\left\langle\mathrm{z}, \mathrm{x}^{\prime}\right\rangle & \langle\mathrm{z}, \mathrm{z}\rangle
\end{array}\right|^{\frac{1}{2}}=\left(\left\|\mathrm{x}^{\prime}\right\|^{2}\|\mathrm{z}\|^{2}\right)^{\frac{1}{2}}=\left\|\mathrm{x}^{\prime}\right\|\|\mathrm{z}\|
$$

serta

$$
\begin{aligned}
& \|\mathrm{y}, \mathrm{z}\|=\left|\begin{array}{cc}
\langle\mathrm{y}, \mathrm{y}\rangle & \langle\mathrm{y}, \mathrm{z}\rangle \\
\langle\mathrm{z}, \mathrm{y}\rangle & \langle\mathrm{z}, \mathrm{z}\rangle
\end{array}\right|^{\frac{1}{2}}=\left|\begin{array}{cc}
\left\langle\mathrm{y}^{\prime}, \mathrm{y}\right\rangle & \left\langle\mathrm{y}^{\prime}, \mathrm{z}\right\rangle \\
\langle\mathrm{z}, \mathrm{y}\rangle & \langle\mathrm{z}, \mathrm{z}\rangle
\end{array}\right|^{\frac{1}{2}}=\left|\begin{array}{cc}
\left\langle\mathrm{y}^{\prime}, \mathrm{y}^{\prime}\right\rangle & \left\langle\mathrm{y}^{\prime}, \mathrm{z}\right\rangle \\
\left\langle\mathrm{z}, \mathrm{y}^{\prime}\right\rangle & \langle\mathrm{z}, \mathrm{z}\rangle
\end{array}\right|^{\frac{1}{2}} \\
& =\left|\begin{array}{cc}
\left\langle\mathrm{y}^{\prime}, \mathrm{y}^{\prime}\right\rangle & 0 \\
0 & \langle\mathrm{z}, \mathrm{z}\rangle
\end{array}\right|^{\frac{1}{2}}=\left(\left\|\mathrm{y}^{\prime}\right\|^{2}\|\mathrm{z}\|^{2}\right)^{\frac{1}{2}}=\left\|\mathrm{y}^{\prime}\right\|^{2}\|\mathrm{z}\|^{2},
\end{aligned}
$$

sehingga dari (3), (4), dan (5) diperoleh:

$$
\cos \theta=\frac{\langle x, y \mid z\rangle}{\|x, z\|\|y, z\|}=\frac{\left\langle x^{\prime}, y^{\prime}\right\rangle\|z\|^{2}}{\left\|x^{\prime}\right\|\left\|y^{\prime}\right\|\|z\|^{2}}=\frac{\left\langle x^{\prime}, y^{\prime}\right\rangle}{\left\|x^{\prime}\right\|\left\|y^{\prime}\right\|}
$$

Hal ini menunjukkan, nilai sudut yang dibangun oleh dua bidang (yang direntang oleh $\mathrm{x}$ dan z serta bidang yang di rentang oleh y dan z) juga dapat dihitung dengan 


$$
\cos \theta=\frac{\langle x, y \mid z\rangle}{\|x, z\|\|y, z\|}=\frac{\left\langle x^{\prime}, y^{\prime}\right\rangle\|z\|^{2}}{\left\|x^{\prime}\right\|\left\|y^{\prime}\right\|\|z\|^{2}}=\frac{\left\langle x^{\prime}, y^{\prime}\right\rangle}{\left\|x^{\prime}\right\|\left\|y^{\prime}\right\|}
$$

Dimana $x^{\prime} \perp z$ dan $y^{\prime} \perp z$ (perhatikan gambar 1).

\section{Kesimpulan}

Dari hasil pembahasan dapat disimpulkan bahwa:

1. Secara umum norm-2 dapat diinterpretasikan sebagai luas jajar genjang yang direntang oleh dua buah vektor.

2. Ruang hasil kali dalam-2 merupakan suatu ruang norm-2,dengan norm-2 yang diinduksi oleh hasil kali dalam-2 (norm-2 yang didefinisikan pada hubungan hasil kali dalam-2 dengan norm2).

3. Nilai sudut yang dibangun oleh dua bidang (yang direntang oleh $x$ dan $z$ serta bidang yang di rentang oleh $y$ dan $z$ dapat dihitung dengan bantuan dua vektor lain yakni $x^{\prime}$ dan $y^{\prime}$ dimana $x^{\prime}$ merupakan proyeksi vector $x$ pada $z$ dan $y^{\prime}$ merupakan proyeksi vector $y$ pada $z$.

4. Sebagai tambahan bahwa, pembaca akan dapat melihat bahwa norm-2 sebanarnya merupakan suatu norm dan hasil kali dalam-2 merupakan suatu hasil kali dalam.

\section{Daftar Pustaka}

1. Batkunde, H., Gunawan, H., Pangalela, Y. E. P., 2013. Bounded linear functional on n-normed spaces of p-summable sequences, AUMB, pp 66-75.

2. Kreyszig E., 1978. Introductory Functional Analysis With Applications, John Wiley and Sons Inc.

3. Gahler S., "Lineare 2-nomierte Raume", Math.Nachr, 1964.pp 1-43

4. Gahler S., 1969. "Untersuchungenuberverallgemeinerte m-metrische Raume", Math.Nachr, pp 229-264

5. Gunawan H., 2010. Onn-normed spaces and n-inner product spaces. Int. J. Math. Math. Sci,

6. Gunawan H., Onn-inner product, n-norms, and the Cauchy-Schwarz Inequality, Sci. Math, Japon, 2010.

7. Gunawan H., \& Mashadi, 2001. On finite dimensional 2-normed spaces, Soochow J. Math, pp 321329.

8. Misiak A., 1989. n-inner product spaces. Math. Nachr, pp 299-319. 